\title{
Local INR correction: justification for a simplified approach
}

\author{
S Craig, K J Stevenson, J M K Dufty, D A Taberner
}

\begin{abstract}
Aims-Errors in reporting International Normalised Ratios (INR) may be corrected by assignment of a System International Sensitivity Index (System ISI). This 57 centre study tests the validity of several procedures for INR correction.

Methods-Prothrombin times of eight lyophilised coumarin calibrants, a lyophilised normal pool calibrant, and eight frozen coumarin plasmas were determined at each centre. The calibrants were calibrated using international reference preparations. The eight frozen coumarin plasmas were calibrated in a four centre international exercise. The relations tested were: (a) the logarithm of local prothrombin time against the logarithm of reference prothrombin time; (b) reference INR against local prothrombin time; and (c) logarithm of reference INR against logarithm of local prothrombin time. These methods were analysed by both linear and orthogonal regression.
\end{abstract}

Results-All system groups required correction, the mean percentage deviation of the uncorrected data from the calibrated values was $19.0 \%$. There was also considerable variation in INR, with the coefficient of variance (CV) ranging from 11.30 to $17.29 \%$. Correction of INR was possible with all methods (CV reduced to $<7 \%$ ). However, only when a plot of the logarithm of local prothrombin time against the logarithm of reference prothrombin time was fitted by orthogonal regression, or a plot of logarithm of reference INR against logarithm of local prothrombin time was fitted by either type of regression analysis, did the best fit line through the calibrant plasmas also pass close to the local mean normal pro-

Conclusions-While INR correction may be achieved by all the above methods, that relating log reference INR to log local prothrombin time by linear regression analysis is the simplest to perform.

(F Clin Pathol 1997;50:783-789)

Thrombosis Reference Centre, Withington Hospital, Manchester, M20 2LR, UK

Correspondence to: Mr Craig.

Accepted for publication 12 June 1997

There have been many studies on the error in (INR) owing to the use of coagulometers. It is possible to reduce this error by the assignment of a System International Sensitivity Index thrombin time.

(System ISI), that is, an ISI specific for the combination or "System" of coagulometer and thromboplastin reagent in local use. This has been shown to be important in correcting instrument specific ISI as well as thromboplastins with an ISI assigned manually. ${ }^{1}$

Clarke et $a l^{2}$ demonstrated that a method based on a simplified WHO calibration using calibrant plasmas could be used in the routine laboratory for correction of INR. This method makes use of orthogonal regression as described in the original WHO protocol. ${ }^{3}$ Further simplifications have emerged that use linear regression instead. For example, Moritz et $a l^{4}$ for example, showed that it was possible to improve interlaboratory precision by performing a local calibration using coumarin plasma calibrants. In their method, the logarithm of the reference INR was plotted against the logarithm of the local prothrombin time. A similar improvement in precision was noted by Kitchen $e t a l^{5}$ in a UK National External Quality Assurance Scheme supplementary exercise, where the reference INR was plotted against the local prothrombin time. In both these methods it is possible to derive a value for the mean normal prothrombin time, removing the
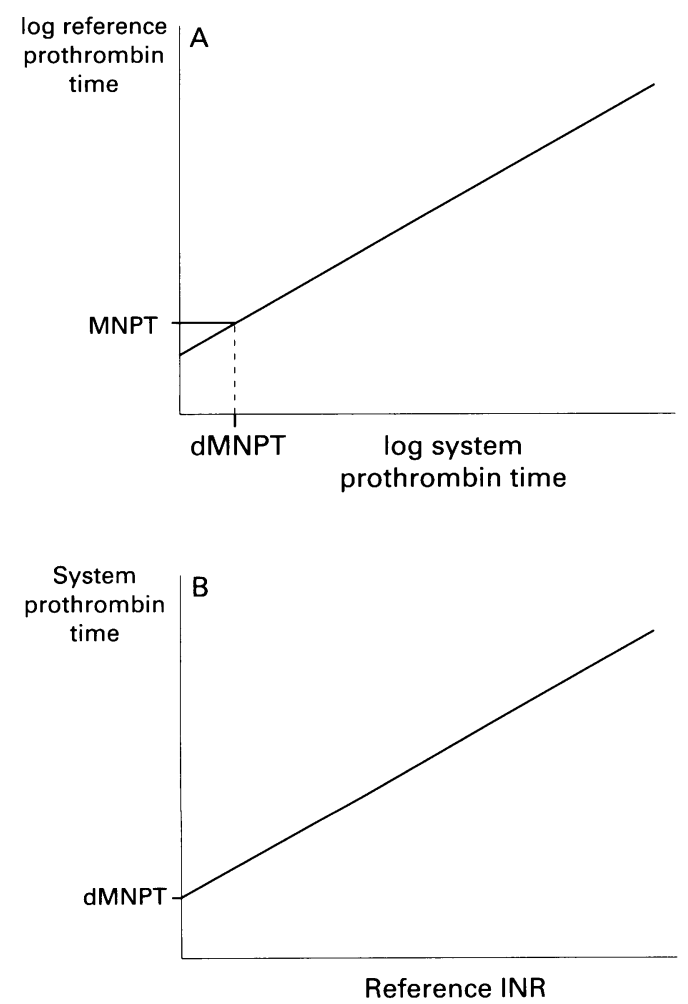
reporting of International Normalised Ratios

Figure 1 Determination of derived mean normal prothrombin time (dMNPT). (A) From a plot of logarithms of System prothrombin time against logarithms of reference prothrombin times. (B) From a plot of reference INR against System prothrombin times. 
Table 1 Systems employed

\begin{tabular}{lll}
\hline Coagulometer & Thromboplastin reagent & $\begin{array}{l}\text { Number of } \\
\text { users }\end{array}$ \\
\hline ACL & IL PT Fibrinogen HS Plus & 21 \\
ACL & $\begin{array}{l}\text { Low opacity Manchester } \\
\text { reagent }\end{array}$ & 12 \\
KC & Manchester reagent & 6 \\
Sysmex & Sysmex PT (CA) & 5 \\
Sysmex & Low opacity Manchester & 3 \\
Sysmex & reagent & 2 \\
Sysmex & Diagen activated & 1 \\
Sysmex & Danchester reagent & 1 \\
Sysmex & Dade In & 1 \\
Coag-a-Mate & Manchester reagent & 2 \\
MLA Electra & Manchester reagent & 2 \\
Hemolab & Low opacity Manchester & 1 \\
$\quad$ Biomerieux & reagent & 57 \\
Total & & \\
\hline
\end{tabular}

Table 2 INR of coumarin calibrant plasmas

\begin{tabular}{llllll}
\hline BCT 441 & RBT 1303 & OBT 79 & RBT 90 & Mean & \multicolumn{1}{l}{$C V$} \\
\hline 2.66 & 2.41 & 2.48 & 2.22 & 2.45 & 6.00 \\
2.94 & 2.56 & 2.69 & 2.54 & 2.76 & 7.90 \\
3.75 & 3.14 & 3.22 & 3.50 & 3.44 & 7.30 \\
2.16 & 1.91 & 1.77 & 1.85 & 1.93 & 7.60 \\
3.64 & 3.14 & 3.36 & 3.16 & 3.39 & 7.10 \\
2.07 & 2.06 & 1.84 & 2.03 & 2.01 & 4.70 \\
3.47 & 3.59 & 3.19 & 3.76 & 3.73 & 14.20 \\
2.71 & 2.46 & 2.27 & 2.76 & 2.63 & 10.00 \\
\hline
\end{tabular}

$\mathrm{CV}$, coefficient of variation.

need for routine laboratories to determine their local value.

The present multicentre study tests the validity of a variety of alternative procedures for local correction of INR. In addition the accuracy of derived mean normal prothrombin time (dMNPT) is assessed.

\section{Methods}

Eight lyophilised coumarin plasmas, one lyophilised normal pool plasma, and eight frozen coumarin plasmas were despatched from the Thrombosis Reference Centre to each of
Table 3 INR of validator plasmas determined at four centres with RBT 90

\begin{tabular}{lllllll}
$\begin{array}{l}\text { Plasma } \\
\text { No }\end{array}$ & Centre 1 & Centre 2 & Centre 3 & Centre 4 & Mean & CV \\
\hline 1 & 2.27 & 2.60 & 2.61 & 2.21 & 2.42 & 8.8 \\
2 & 1.87 & 2.00 & 2.30 & 1.91 & 2.02 & 9.5 \\
3 & 2.25 & 2.40 & 2.41 & 2.51 & 2.39 & 4.4 \\
4 & 2.58 & 2.59 & 2.79 & 3.07 & 2.76 & 8.3 \\
5 & 2.69 & 2.70 & 3.16 & 2.80 & 2.84 & 7.8 \\
6 & 2.78 & 2.74 & 3.06 & 2.74 & 2.83 & 5.6 \\
7 & 3.10 & 3.22 & 3.48 & 3.53 & 3.33 & 6.2 \\
8 & 3.00 & 3.31 & $4.56^{\star}$ & 3.29 & 3.20 & 5.4
\end{tabular}

*Excluded from calculation of the mean. CV, coeffecient of variation.

57 participating laboratories. The frozen coumarin plasmas were transported in dry ice by overnight carrier.

Coumarin plasmas were obtained from patients stabilised on oral anticoagulant therapy. Eight donations were dispensed in $0.5 \mathrm{ml}$ aliquots for freeze drying. Eight further donations were dispensed in $0.5 \mathrm{ml}$ aliquots and frozen at $-80^{\circ} \mathrm{C}$. In addition, a normal plasma pool was prepared from 13 donations and dispensed in $0.5 \mathrm{ml}$ aliquots for lyophilisation.

Freeze drying was performed according to a protocol devised at the Thrombosis Reference Centre. Briefly, this consisted of a primary drying phase of $\sim 48$ hours with a condenser temperature of less than $-65^{\circ} \mathrm{C}$, followed by a secondary drying phase of 24 hours over phosphorus pentoxide. The vials were stoppered in vacuo. All plasmas were protected during the freeze drying process by the addition of sucrose $(10.9 \mathrm{mmol} / \mathrm{l})$ and glycine $(58.4 \mathrm{mmol} / \mathrm{l})$. The $\mathrm{pH}$ of the plasmas was maintained at physiological level by the addition of HEPES buffer $(266.4 \mathrm{mmol} / \mathrm{l})$.

All calibrant plasmas were calibrated manually, in duplicate, by five scientific officers at the Thrombosis Reference Centre using the cur-
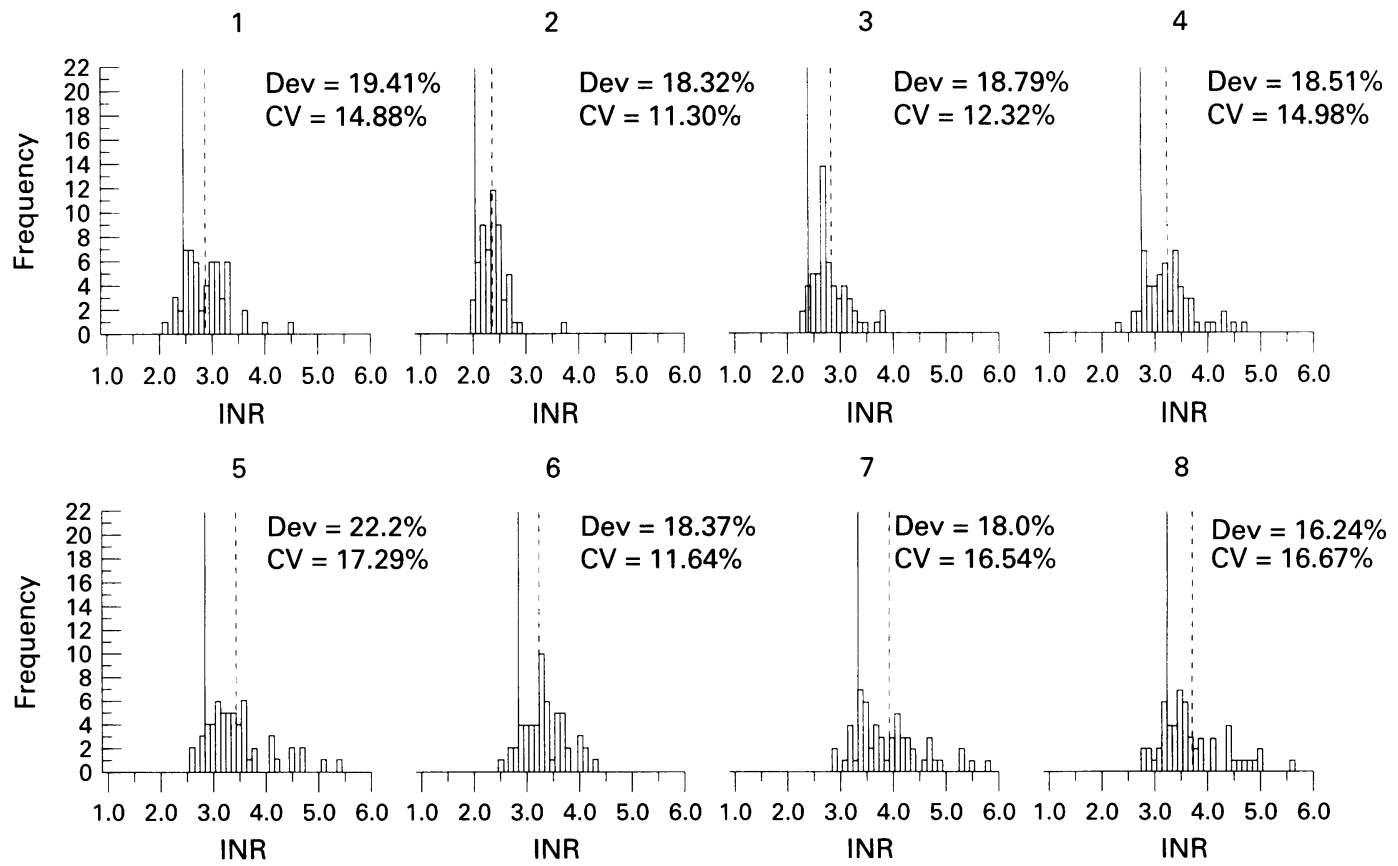

Figure 2 Frequency distributions of uncorrected INR values for eight frozen coumarin plasmas. The solid line represents the calibrated value obtained in a four centre calibration exercise and the dotted line represents the mean of the uncorrected INR obtained at the 57 participating laboratories. The percentage deviation of the System value from the manual calibrated value and the coefficient of variance $(C V)$ of the distribution are given. 


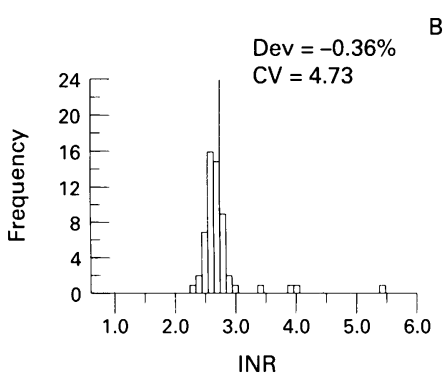

BCT 441

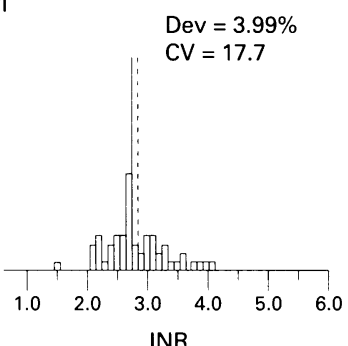

RBT 1303
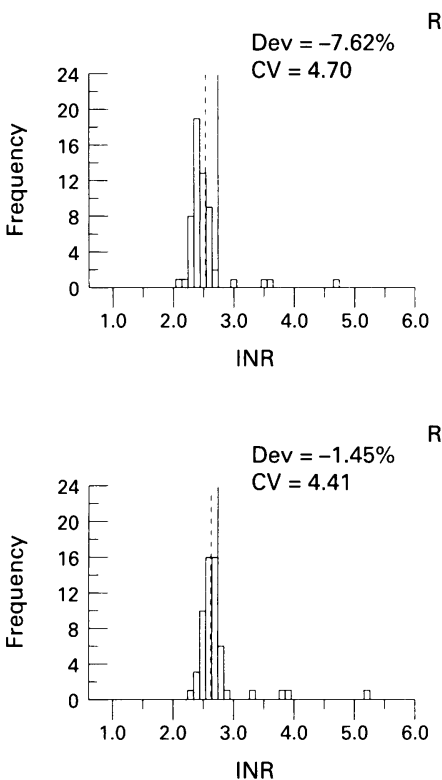

RBT 90

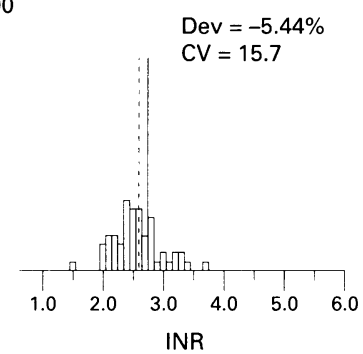

OBT 79
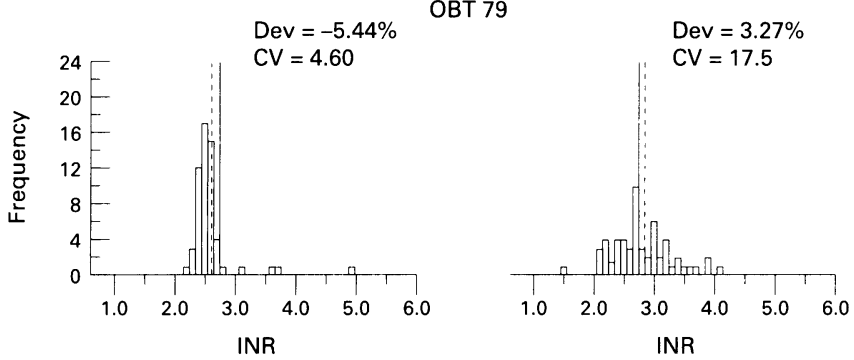

Figure 3 Frequency distributions of INR for frozen coumarin plasma 4, corrected by methods $A$ (left) and $B$ (right). The solid line represents the calibrated value and the dotted line represents the mean of the corrected INR. The percentage deviation of the System value from the manual calibrated value and the coefficient of variance $(C V)$ of the distribution are given.

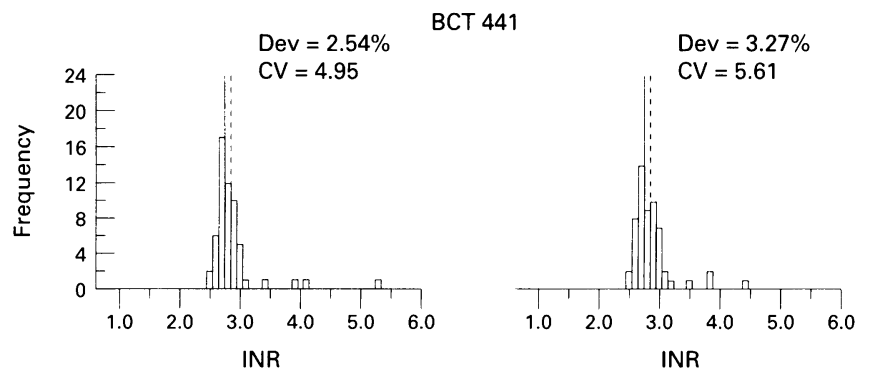

RBT 1303
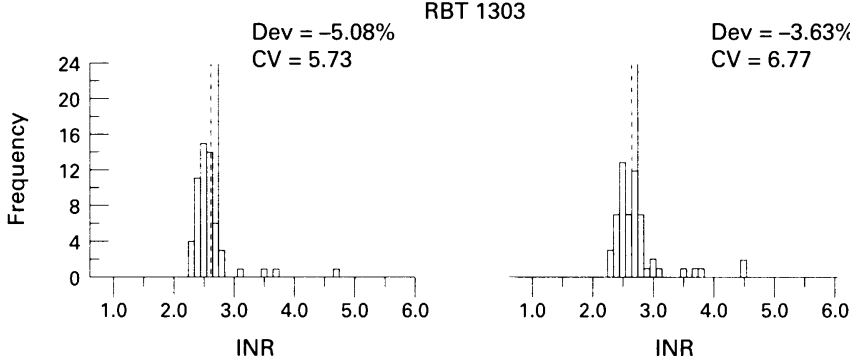

RBT 90

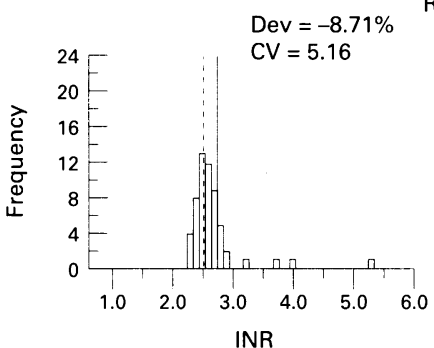

Dev $=-7.26 \%$

$\mathrm{CV}=4.30$

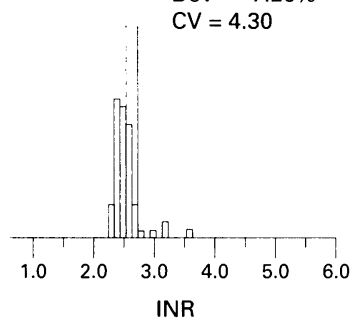

OBT 79

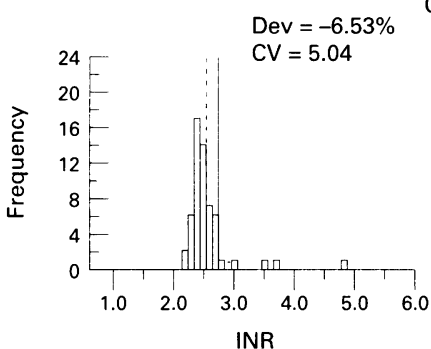

Dev $=-9.07 \%$

$\mathrm{CV}=5.18$

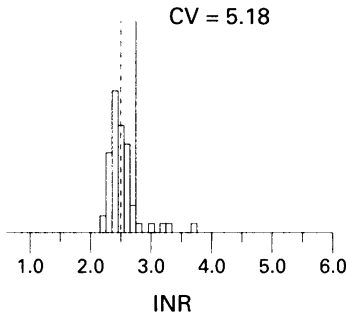

Figure 4 Frequency distributions of INR for frozen coumarin plasma 4 , corrected by methods $C$ (left) and D (right). The solid line represents the calibrated value and the dotted line represents the mean of the corrected INR. The percentage deviation of the System value from the manual calibrated value and the coefficient of variance $(C V)$ of the distribution are given.

rent WHO International Reference Preparations RBT 90 (rabbit, plain) and OBT 79 (bovine, combined). In addition, BCT 441 (human, plain), a reference preparation of the International Committee for Standards in Haematology, and RBT 1303 (rabbit, plain), our own in-house reagent, were used. RBT 1303 had been calibrated previously against BCT 441 and RBT 90. INR were calculated using the mean normal prothrombin time determined in the international calibration of the reference thromboplastins.

The frozen coumarin plasmas were calibrated internationally in a four centre exercise using RBT 90. Prothrombin times of each plasma were determined manually, in triplicate, at each of the four centres. The INR were calculated using the ISI and mean normal prothrombin time established in the international calibration of RBT 90. These plasmas were used to test the validity of the local calibration (validator plasmas).

The 57 centres were asked to determine the prothrombin time, in quadruplicate, of each plasma using their local combination of coagulometer and thromboplastin reagent and to return their results for analysis. In addition, each centre provided a mean normal prothrombin time based on calculation of the geometric mean of at least 20 normal plasmas.

For each of the frozen coumarin plasmas an INR frequency distribution of the uncorrected data was constructed. The difference between the calibrated INR value and the mean INR value of those distributions were calculated together with the coefficient of variation (CV).

For each laboratory the slope of the line calibrant plasmas and the local System mean normal prothrombin time to the calibrated valrelating the local System results for the 
Table 4 Comparison of local MNPT with derived MNPT. RBT 90 used as reference

\begin{tabular}{|c|c|c|c|c|c|c|c|}
\hline & Local MNPT & C & $D$ & $E$ & $F$ & $G$ & $H$ \\
\hline ACL + IL PT Fibrinogen HS Plus & 13.28 & $15.37(15.7)$ & $12.28(-7.5)$ & $12.13(-8.7)$ & $17.08(28.6)$ & $15.37(15.7)$ & $16.07(21.0)$ \\
\hline $\mathrm{ACL}+$ Manchester reagent & 11.89 & $13.79(16.0)$ & $10.25(-13.8)$ & $14.14(18.9)$ & $15.08(26.8)$ & $13.79(16.0)$ & $13.93(17.2)$ \\
\hline $\mathrm{KC}+$ Manchester reagent & 13.76 & $16.38(19.0)$ & $13.27(-3.5)$ & $16.60(20.6)$ & $14.48(5.2)$ & $16.38(19.0)$ & $16.51(20.0)$ \\
\hline Sysmex + Sysmex PT (CA) & 15.02 & $15.85(5.5)$ & $11.31(-24.6)$ & $14.72(-2.0)$ & $16.90(12.5)$ & $15.85(5.5)$ & $16.12(7.3)$ \\
\hline Others & 12.90 & $14.49(12.3)$ & $11.00(-14.7)$ & $14.47(12.2)$ & $15.71(21.8)$ & $14.49(12.3)$ & $14.66(13.6)$ \\
\hline All groups & 13.11 & $14.99(14.3)$ & $11.58(-11.7)$ & $13.78(5.1)$ & $16.37(24.9)$ & $14.99(14.3)$ & $15.35(17.1)$ \\
\hline
\end{tabular}

Value in brackets are the percentage deviation of the dMNPT from the local MNPT.

Table 5 Comparison of local MNPT with derived MNPT. OBT 79 used as reference

\begin{tabular}{|c|c|c|c|c|c|c|c|}
\hline & Local MNPT & $C$ & $D$ & $E$ & $F$ & $G$ & $H$ \\
\hline ACL + IL PT Fibrinogen HS Plus & 13.28 & $14.53(9.4)$ & $14.73(10.9)$ & $12.66(-4.7)$ & $14.54(9.5)$ & $14.54(9.5)$ & $14.78(11.3)$ \\
\hline $\mathrm{ACL}+$ Manchester reagent & 11.89 & $13.14(10.5)$ & $11.95(0.5)$ & $13.56(14.0)$ & $14.06(18.3)$ & $13.14(10.5)$ & $13.23(11.3)$ \\
\hline $\mathrm{KC}+$ Manchester reagent & 13.76 & $15.58(13.2)$ & $15.24(10.8)$ & $15.46(12.4)$ & $16.48(19.8)$ & $15.58(13.2)$ & $15.75(14.5)$ \\
\hline Sysmex + Sysmex PT (CA) & 15.02 & $15.09(0.5)$ & $13.28(-11.6)$ & $14.26(-5.1)$ & $15.49(3.1)$ & $15.09(0.5)$ & $15.25(1.5)$ \\
\hline Others & 12.90 & $13.81(7.1)$ & $12.71(-1.5)$ & $13.77(6.7)$ & $14.75(14.3)$ & $13.81(7.1)$ & $13.99(8.4)$ \\
\hline All groups & 13.11 & $14.23(8.5)$ & $13.61(4.0)$ & $13.54(3.3)$ & $14.78(12.7)$ & $14.24(8.8)$ & $14.42(10.0)$ \\
\hline
\end{tabular}

Values in brackets are the percentage deviation of the dMNPT from the local MNPT.

Table 6 Comparison of local MNPT with derived MNPT. BCT 441 used as reference

\begin{tabular}{|c|c|c|c|c|c|c|c|}
\hline & Local MNPT & $C$ & $D$ & $E$ & $F$ & $G$ & $H$ \\
\hline$\overline{\text { ACL + IL PT Fibrinogen HS Plus }}$ & 13.28 & $12.61(-5.0)$ & $14.16(6.6)$ & $10.30(-22.4)$ & $11.81(-11.1)$ & $12.62(-5.0)$ & $12.84(-3.3)$ \\
\hline $\mathrm{ACL}+$ Manchester reagent & 11.89 & $11.51(-3.2)$ & $11.45(-3.7)$ & $11.73(-1.3)$ & $12.10(-1.8)$ & $11.51(-3.2)$ & $11.57(-2.7)$ \\
\hline $\mathrm{KC}+$ Manchester reagent & 13.76 & $13.59(-1.2)$ & $14.65(6.5)$ & $13.19(-4.1)$ & $13.95(1.4)$ & $13.59(-1.2)$ & $13.70(-0.4)$ \\
\hline Sysmex + Sysmex PT (CA) & 15.02 & $13.25(-11.8)$ & $12.80(-14.8)$ & $12.33(-17.9)$ & $12.96(-13.7)$ & $13.26(-11.7)$ & $13.35(-11.1)$ \\
\hline Others & 12.90 & $12.07(-6.4)$ & $12.22(-5.3)$ & $13.42(4.0)$ & $12.61(-2.2)$ & $12.07(-6.4)$ & $12.21(-5.3)$ \\
\hline All groups & 13.11 & $12.41(-5.3)$ & $13.08(-0.2)$ & $11.40(-13.0)$ & $12.39(-5.5)$ & $12.42(-5.2)$ & $12.56(-4.2)$ \\
\hline
\end{tabular}

Values in brackets are the percentage deviation of the dMNPT from the local MNPT.

Table 7 Comparison of local MNPT with derived MNPT. RBT 1303 used as reference

\begin{tabular}{|c|c|c|c|c|c|c|c|}
\hline & Local MNPT & $C$ & $D$ & $E$ & $F$ & $G$ & $H$ \\
\hline ACL + IL PT Fibrinogen HS Plus & 13.28 & $13.39(0.8)$ & $17.20(29.5)$ & $9.51(-28.4)$ & $14.64(10.2)$ & $13.46(1.35)$ & $14.13(6.4)$ \\
\hline $\mathrm{ACL}+$ Manchester reagent & 11.89 & $12.23(2.9)$ & $14.48(21.8)$ & $12.37(4.0)$ & $13.03(9.6)$ & $12.24(2.9)$ & $12.33(3.7)$ \\
\hline $\mathrm{KC}+$ Manchester reagent & 13.76 & $14.46(5.1)$ & $18.39(33.6)$ & $14.12(2.6)$ & $14.97(8.1)$ & $14.47(4.9)$ & $14.58(6.0)$ \\
\hline Sysmex + Sysmex PT (CA) & 15.02 & $14.06(-6.4)$ & $15.96(6.3)$ & $12.30(-18.1)$ & $14.92(-0.7)$ & $14.09(-6.2)$ & $14.41(-4.1)$ \\
\hline Others & 12.90 & $12.84(-0.5)$ & $15.36(19.1)$ & $12.57(-2.6)$ & $12.69(-1.6)$ & $12.86(-0.3)$ & $13.01(0.9)$ \\
\hline All groups & 13.11 & $13.19(0.6)$ & $16.22(23.7)$ & $11.54(-12.0)$ & $14.11(7.6)$ & $13.23(0.9)$ & $13.57(3.5)$ \\
\hline
\end{tabular}

Values in brackets are the percentage deviation of the dMNPT from the local MNPT.

ues obtained with each of the reference thromboplastins and the reference mean normal prothrombin time was derived by orthogonal regression. This method (method $\mathrm{A})$ is an abbreviated version of the WHO recommended procedure that has been used successfully in the UK. ${ }^{1}$ This simplified calibration was modified further by using linear, rather than orthogonal, regression to calculate the slope (method B), by replacing mean normal prothrombin time with lyophilised normal pool plasma, and using both orthogonal regression (method C), and linear regression (method D). In addition, the equation of the line relating local prothrombin times to INR assigned using each of the reference thromboplastins was calculated by orthogonal (method E), and linear regression (method F). Finally, the System ISI was calculated by repeating the latter two methods but using logarithms of the local prothrombin times and of the reference INR, again using both orthogonal regression (method $G$ ), and linear regression (method $\mathrm{H}$ ).

To test the effectiveness of the correction procedures the INR frequency distributions of the frozen coumarin plasmas, following correction by the above methods, were constructed for each reference thromboplastin. The difference between the calibrated INR value and the mean INR of the corrected data was calculated, together with the CV. In order to reduce the influence of outliers on the description of the frequency distributions, a series of iterations were performed in which the mean and standard deviation of the distribution were calculated and data points excluded that lay outside the mean + or -3 SD.

In addition, in those methods where the lyophilised normal pool calibrant value replaced the locally calculated mean normal prothrombin time in calculation of the slope of the calibration line, a derived mean normal prothrombin time (dMNPT) was obtained by reading from the calibration line the point on the System axis equivalent to the mean normal prothrombin time of the reference (fig 1); for instance, in fig $1 \mathrm{~A}$ the dMNPT of the System is the value on the $\mathrm{x}$ axis equivalent to the mean normal prothrombin time of the reference. In fig $1 \mathrm{~B}$ the dMNPT of the System is the value on the $\mathrm{Y}$ axis equivalent to an INR value of 1.0 with the reference thromboplastin. The mean percentage deviation of the dMNPT from the locally determined value was also calculated.

\section{Results}

The various combinations of coagulometer and thromboplastin reagents used in the exercise are shown in table 1. All major instruments and reagents in use in the UK were represented. All 


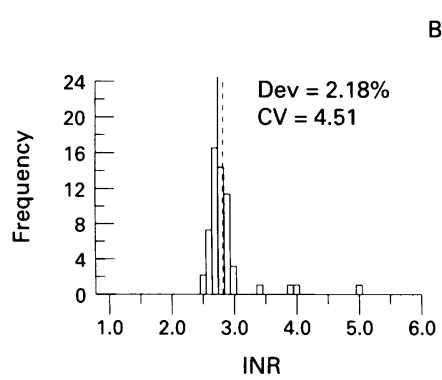

BCT 441

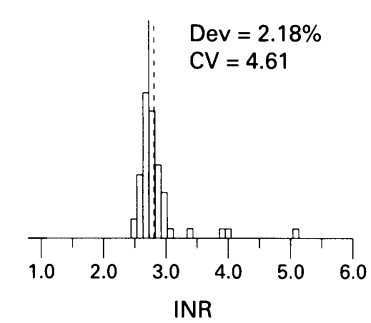

RBT 1303
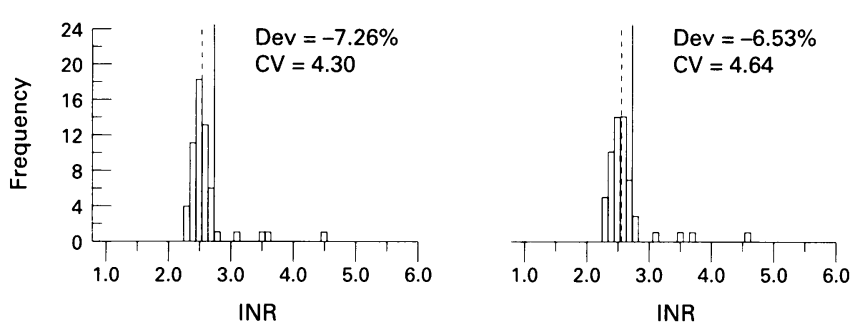

RBT 90
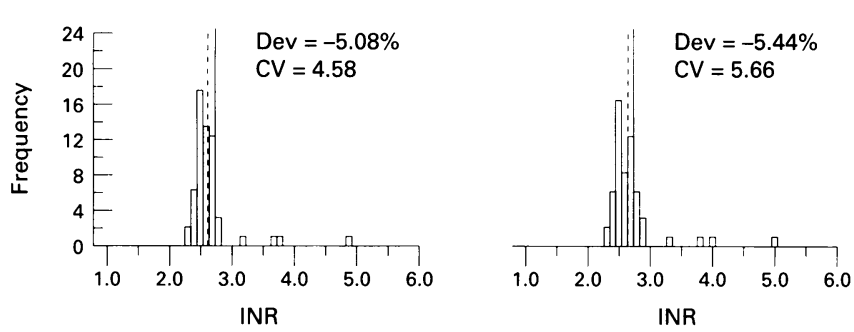

OBT 79
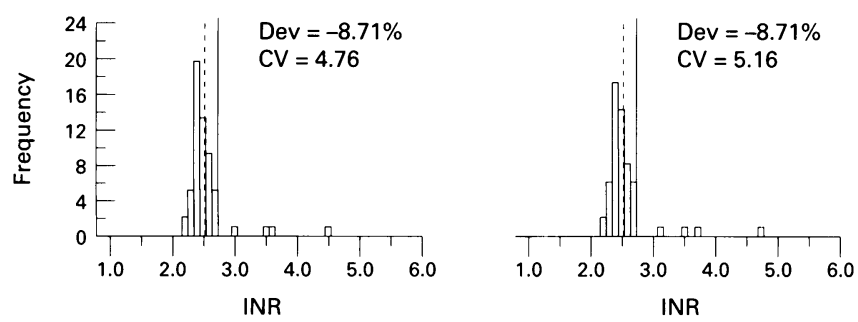

Figure 5 Frequency distributions of INR for frozen coumarin plasma 4, corrected by methods $E$ (left) and $F$ (right). The solid line represents the calibrated value and the dotted line represents the mean of the corrected INR. The percentage deviation of the System value from the manual calibrated value and the coefficient of variance $(C V)$ of the distribution are given.

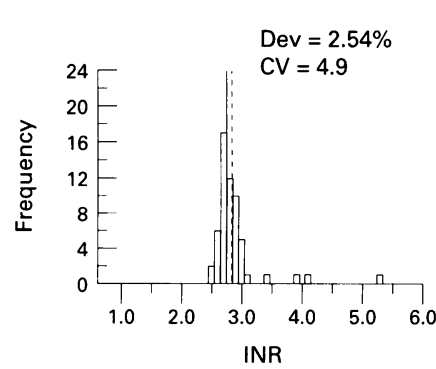

BCT 441
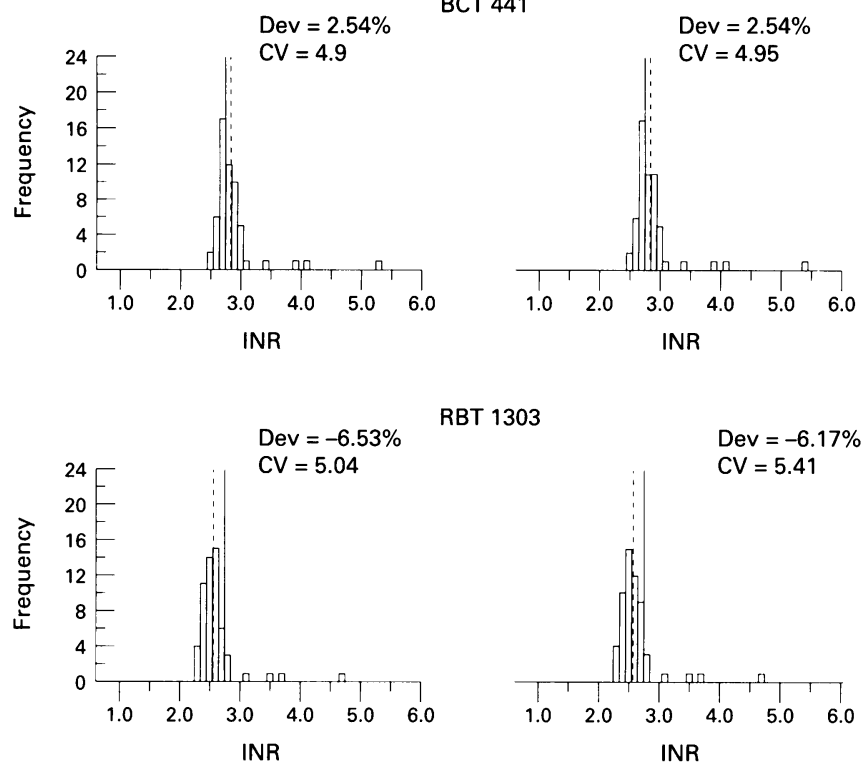

RBT 1303
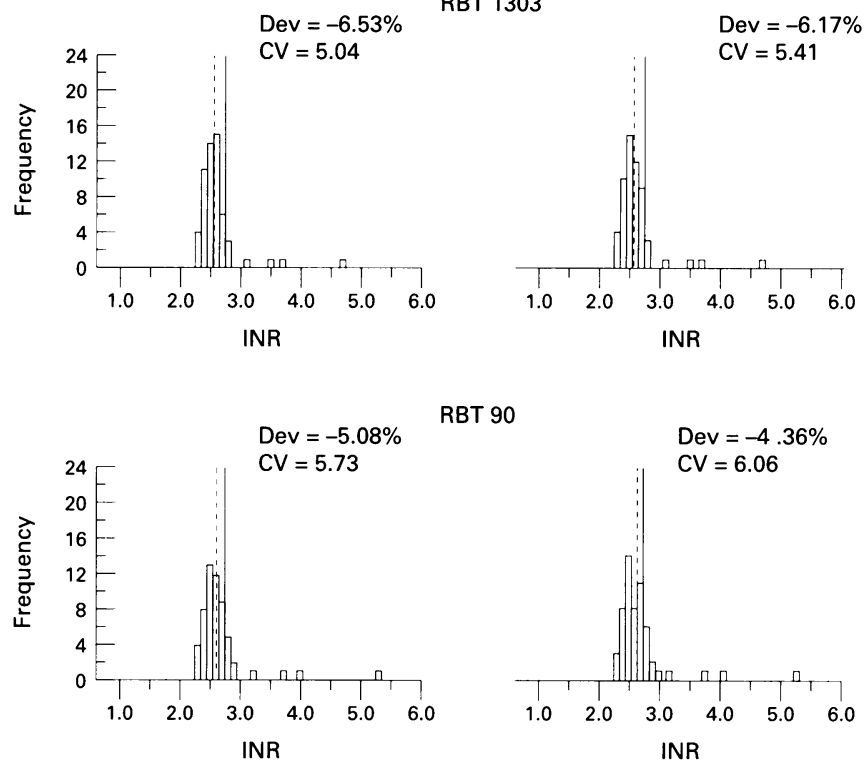

RBT 90
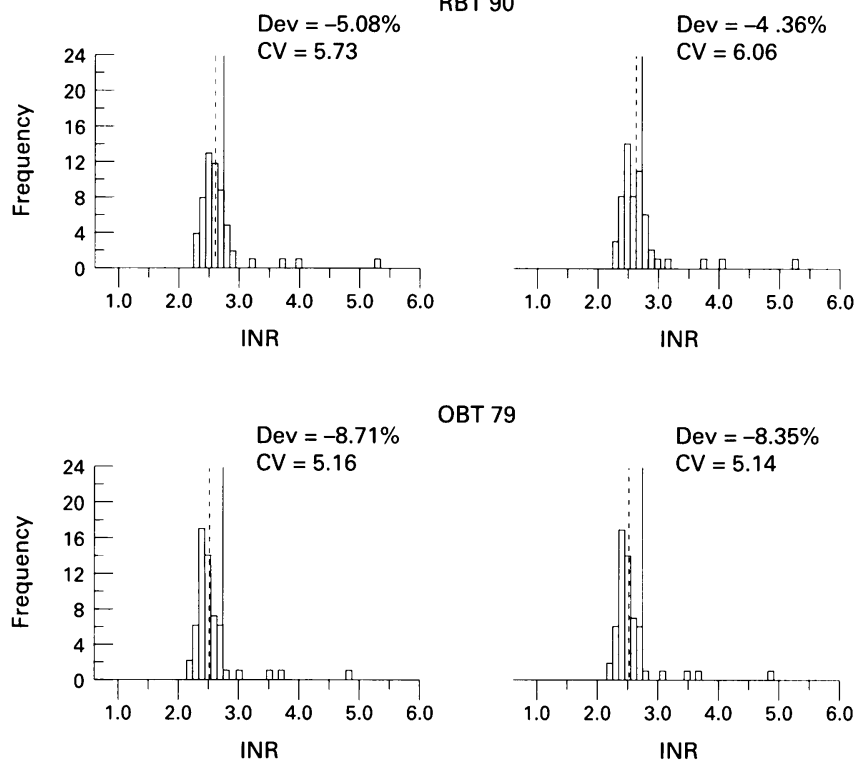

OBT 79

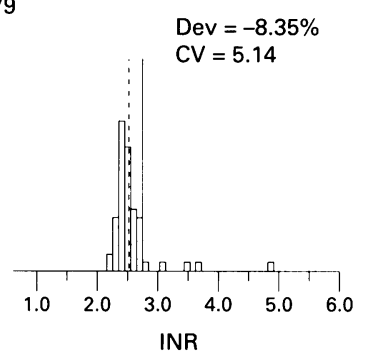

Figure 6 Frequency distributions of INR for frozen coumarin plasma 4, corrected by methods $G$ (left) and $H$ (right). The solid line represents the calibrated value and the dotted line represents the mean of the corrected INR. The percentage deviation of the System value from the manual calibrated value and the coefficient of variance $(C V)$ of the distribution are given.
Systems used by fewer than five centres (13 in all) were grouped as "other" for the purposes of subsequent data manipulation. Of the 57 centres, 26 used an instrument specific ISI, 21 of these being ACL coagulometers with IL PT Fibrinogen HS Plus reagent and five being Sysmex coagulometers with Sysmex PT (CA) reagent. Of the remaining 31 centres, 29 used a manually assigned ISI and two used a value assigned for photo-optic instruments.

The INR values obtained for the calibrant plasmas using RBT 90, OBT 79, BCT 441, and RBT 1303 are given in table 2. The INR values obtained with RBT 90 in the four centre international calibration of the frozen coumarin validator plasmas are shown in table 3 . The coefficients of variation are also given.

The uncorrected INR values obtained for the eight frozen coumarin plasmas by the 57 participating laboratories showed considerable variation (fig 2). Coefficients of variation ranged from $11.30 \%$ to $17.29 \%$. The consensus mean INR values were consistently higher than those obtained manually with RBT 90 . Figures 3-6 illustrate the correction obtained by each of the calculation methods using plasma number 4 as an example. All the calculation methods, except method B (fig 3) led to a marked improvement in accuracy and precision. Although method B led to improvement in accuracy with all thromboplastins, except RBT 1303, there was no improvement in precision with any reagent.

When the lyophilised normal pool calibrant was included in the calculation instead of local mean normal prothrombin time, a dMNPT was obtained as shown in tables 4-7. In general, the dMNPT showed good agreement 
with the locally determined mean normal prothrombin time only when BCT 441 or RBT 1303 were used as reference thromboplastins in methods $\mathrm{C}, \mathrm{G}$, and $\mathrm{H}$. In all System groups, dMNPTs were within $6.4 \%$ of the locally calculated mean normal prothrombin time with the exception of Sysmex + Sysmex PT (CA) when BCT 441 was the reference thromboplastin. If methods D, E, and F were used to determine a System ISI, the dMNPT of some Systems differed markedly from the local mean normal prothrombin time. The use of either OBT 79 or RBT 90 as reference thromboplastin in the System ISI calibration led to marked differences in the dMNPT of some Systems from the calculated mean normal prothrombin time, regardless of the method used.

\section{Discussion}

This study is based on current practice in the UK, with 57 participating centres representing the methods and reagents in common, routine use. It has shown that after 30 years of attempts to standardise the prothrombin time there is still serious cause for alarm. For example, plasma 1 had a calibrated manual INR value of 2.4, but results were obtained between 2.1-4.5, spanning almost the entire therapeutic range. This unsatisfactory situation exists despite regular quality assurance surveys. A correction step has been shown to be essential to bring the local coagulometer/reagent combination back to concordance with the WHO system with its requirement for manual testing. ${ }^{6}$ Several approaches have been used to perform this correction and the validity of these are tested in this study.

Undoubtedly, one component of variation is the growing dependence on coagulometers, which can influence the manufacturers' assigned reagent ISI in an unpredictable fashion. ${ }^{7}$ In 1989, D'Angelo et $a l^{8}$ demonstrated that ACL and Koagulab instruments overestimated the INR in comparison with the manual technique. Our findings support these observations with each System group overestimating INR by between 10 and $25 \%$. This was the case for thromboplastins with instrument specific ISI as well as those with only a manually assigned value. The fact that the majority of centres overestimated the INR is not surprising for those centres using manually assigned ISI. In this study, however, the largest deviations from the value calibrated internationally were seen in the ACL coagulometer and IL PT Fibrinogen HS Plus reagent group that did use an instrument specific ISI. We have no reason to doubt the values assigned to the frozen coumarin plasmas in the international exercise and, therefore, can only conclude that there is some error in the ISI assigned to this group.

It is important to validate the corrected System ISI by the use of a set of individual patient plasmas. Frozen coumarin plasmas were preferred as it was shown in a previous study that the INR of such plasmas did not vary from that found in the original freshly drawn sample. ${ }^{9} \mathrm{On}$ the other hand, the INR values of lyophilised plasmas were significantly different from those of fresh plasmas and varied according to the thromboplastin used, making their use as validators inappropriate. ${ }^{10}$ Ideally, frozen coumarin plasmas should be used as calibrants, as well as validators, but currently this is impractical because of the expense of distribution. The factor $\mathrm{V}$ and fibrinogen levels in the calibrant plasmas used in this study were all tested and shown to be within the normal range and, therefore, should not influence the INR determined with different reagents as has been suggested. ${ }^{11}$

The present study demonstrates clearly that all the approaches used can lead to improvements in reporting INR with the exception of method $B$, which is an abbreviation of the WHO protocol, relating the logarithm of System prothrombin times obtained for the calibrant plasma including the mean normal prothrombin time to the logarithm of reference prothrombin time by linear regression analysis. This is probably because the linear regression method is not robust enough to deal with the situation where the mean normal prothrombin time may not fall on the calibration line. This was the case particularly with RBT 1303.

The calibration line only passed close to the locally determined mean normal prothrombin time if methods $\mathrm{C}, \mathrm{G}$, or $\mathrm{H}$ were used with BCT 441 or RBT 1303 as the reference thromboplastin. As all of the routine reagents were plain thromboplastins it is not surprising that the use of OBT 79 gave rise to a calibration line that did not pass through the mean normal prothrombin time for both reagents. The poor agreement between dMNPT and local calculated mean normal prothrombin time with RBT 90 was a little surprising, however, as this reagent is not only a plain thromboplastin but also of the same species of origin as most of the thromboplastins used in this study. It is possible that the calibration line obtained with RBT 90 as the reference thromboplastin may be influenced by the lyophilisation of the calibrant plasmas. A lyophilised plasma was included in the international calibration of RBT $90 .{ }^{12} \mathrm{~A}$ higher INR value was obtained with RBT 90 than with the other reference thromboplastins tested. If INR levels at values outside the range of the calibrant plasmas are to be accurate, it is important that the line of best fit, however it is derived, should pass through, or near to, the mean normal prothrombin time for both reference and System thromboplastin. ${ }^{13}$

Theoretically, as there are errors in determining the reference INR values as well as the prothrombin times, orthogonal regression should be used. However, the use of linear regression does not seem to affect the result adversely, as method $\mathrm{H}$ gave a result similar to method G. As linear regression is more accessible than orthogonal regression, being available on a number of commercial statistical packages, this would seem to be the preferred method. Another advantage of this method is that local determination of a mean normal prothrombin time is not required. Often, the calculation of a mean normal prothrombin time is difficult owing to the limited availability of normal volunteers. 
In conclusion, this study demonstrates that a local calibration of System ISI improves reporting of INR. Three of the test methods produced improvements in accuracy and precision of the INR and permitted the derivation of an accurate mean normal prothrombin time when BCT 441 or RBT 1303 were used to determine the reference values. These were method C, an abbreviation of the WHO method, using orthogonal regression analysis to relate logarithm of System to reference prothrombin times; method $\mathrm{G}$, using orthogonal regression analysis to relate logarithm of reference INR to logarithm of System prothrombin times; and method $\mathrm{H}$, using linear regression analysis to relate logarithm of reference INR to logarithm of System prothrombin times. Method $\mathrm{H}$ would seem to be the simplest and most convenient of these for most routine laboratories to use.

The authors are most grateful to all the participating centres and to the laboratories that performed the calibration exercise.

1 Stevenson KJ, Craig S, Dufty JMK, Taberner DA. System ISI calibration: a universally applicable scheme is only pos sible when coumarin plasma calibrants are used. $\mathrm{Br} \mathcal{F} \mathrm{Hae}$ matol 1997;96:435-41.

2 Clarke K, Taberner DA, Thomson JM, Morris JA, Poller L. Assessment of value of calibrated lyophilised plasmas to determine International Sensitivity Index for coagulometers. F Clin Pathol 1992;45:58-60.

3 WHO Expert Committee on Biological Standardisation. 33rd Report. WHO Techn Rep Ser 1983;687:81-105.
4 Moritz B, Lang H. AK calibrants. A step forward in the standardisation of the INR. Ann Haematol 1995; 70(Suppl):A27.

5 Kitchen S, Jennings I, Woods TAL, Preston FE. Local calibration for INR determination: results of a 349 laboratory collaborative study. Br f Haematol 1996;93(Suppl 2): 11 .

6 Houbouyan LL, Goguel AF. Procedure of reference calibrated plasmas for prothrombin time standardisation: data from French interlaboratory surveys. Thromb Haemost 1993;69:663.

7 Poggio M, van den Besselaar AMHP, van der Velde EA, Bertina RM. The effect of some instruments for prothrombin time testing on the International Sensitivity Index (ISI) of two rabbit tissue thromboplastin reagents. Thromb Haemost 1989;62:868-74

8 D'Angelo A, Seveso MP, D'Angelo SV, Gilardoni $\mathrm{F}$, Macagni A, Manotti C, et al. Comparison of two automated coagulometers and the manual tilt tube method for the determination of prothrombin time. Am $\mathrm{f}$ Clin Pathol 1989;92:321-8.

9 Craig S, Dufty JMK, Stevenson KJ, Goodman L, Leeming DR, Taberner DA. The effect of freezing and freeze-drying coumarinised plasma on International Normalised Ratio. Blood Coagul Fibrinolysis 1995;6:177.

10 Goodman L, Sidebotham JA, Taberner DA. Sensitivity of thromboplastins to freezing- and freeze drying-induced alterations in coumarin plasmas. Blood Coagul Fibrinolysis 1996;7:11-2

11 Tripodi A, Chantarangkul V, Akkawat B, Clerici M, Mannucci PM. A partial factor V deficiency in anticoagulated lyophilised plasmas has been identified as a cause of the International Normalised Ratio discrepancy in the External Quality Assessment Scheme. Thromb Res 1995;78: 283-92.

12 van den Besselaar AMHP. Multi-centre study of replacement of the International Reference Preparation for thromboplastin, rabbit, plain. Thromb Haemost 1993;70: 794-9

13 Tomenson JA. A statisticians independent viewpoint. In: van den Besselaar AMHP, Gralnick HR, Lewis SM, eds. Thromboplastin and anticoagulant control. Boston: Martinus Nijhoff, 1984:87-108. 